\title{
Editorial
}

\section{Civil Engineering Applications of Polymer Composites}

\author{
Gonzalo Martínez-Barrera, ${ }^{1}$ Osman Gencel, ${ }^{2}$ and João M. L. Reis ${ }^{3}$ \\ ${ }^{1}$ Laboratorio de Investigación y Desarrollo de Materiales Avanzados (LIDMA), Facultad de Química, \\ Universidad Autónoma del Estado de México, Km 12 de la Carretera Toluca-Atlacomulco, 50200 San Cayetano, MEX, Mexico \\ ${ }^{2}$ Civil Engineering Department, Faculty of Engineering, Bartin University, 74100 Bartin, Turkey \\ ${ }^{3}$ Theoretical and Applied Mechanics Laboratory (LMTA), Universidade Federal Fluminense, 24220 Niterói, RJ, Brazil \\ Correspondence should be addressed to Gonzalo Martínez-Barrera; gonzomartinez02@yahoo.com.mx
}

Received 28 December 2015; Accepted 29 December 2015

Copyright (C) 2016 Gonzalo Martínez-Barrera et al. This is an open access article distributed under the Creative Commons Attribution License, which permits unrestricted use, distribution, and reproduction in any medium, provided the original work is properly cited.

Researches have been done in order to improve performance of concrete and structures. As we know, polymers provide huge opportunities for the advancement of materials research, the improvement of material property, and the strengthening of structures damaged. In this respect, recent developments about polymer composites and their technological developments in civil engineering are aimed in this issue.

Topics include recovery and modification of waste and recycled tire polymer fibers and their use as reinforcements of concrete. A novel proposal based on the modification of the physicochemical properties of the waste automotive tire particles, by using gamma irradiation, shows that improvements on the mechanical properties depend of gamma irradiation as well as concentration and size of waste tire particles. Another study shows that PP fibers can be substituted with higher amount of recycled tire polymer fibers obtaining concrete with similar shrinkage behavior. Such applications would contribute to solving the problem of waste tire disposal. Waste materials from Tetra Pak packages have been used as reinforcement of polymer concrete elaborated with unsaturated polyester resin and silica sand and submitted to gamma irradiation. The results show improvements on both compressive strength and flexural strength, as well as modulus of elasticity when polymer concrete is irradiated.

Other studies are concerned with latex polymer cementitious composites (LCCs), for example, polypropylene fibers used in cementitious composites containing nanosilica and styrene butadiene latex polymer, where the addition of nanosilica significantly affected the bond strength and interfacial toughness between macro-PP fiber and cementitious composites. Synthesis of polymers for stabilization of minerals, for example, synthesis of high molecular weight polyacrylamide (PAM) and its application as sand stabilizer: the results showed that the use of high molecular weight polymer gave excellent mechanical and thermal stability to the polymer-sand composite. Other applications of acrylic resins can be found in the paper about environmental impacts on the strength parameters of mineral-acrylic facade panels, elaborated with natural minerals produced from bauxite (aluminium hydroxides, ATH) and from acrylic resin (polymethyl methacrylate, PMMA) and subjected to aging process in conditions of high temperature, high relative humidity, freeze-thaw cycles, and UV radiation.

Studies have been carried out by using fiber reinforced polymer (FRP) materials; as we know, FRP materials have advantages such as corrosion resistance, light weight, machinability, workability, and high strength. The shear capacities of concrete beams reinforced with glass fiber reinforced polymer (GFRP) plates as shear reinforcement have been studied. Also experimental assessment on the flexural bonding performance of concrete beam with GFRP reinforces bar under repeated loading. Moreover, structural behavior of concrete columns internally reinforced with glass fiber reinforced plastic (GFRP) rebars shows that the amount of GFRP longitudinal and lateral reinforcement slightly affects the column strength. another study is focused on the behavior of full-scale porous GFRP barrier under blast loads; a discontinuous (porous) barrier composed by precast concrete reinforced with GFRP bars was studied to take advantage of electromagnetic and mechanical properties as well as mitigating blast shock waves. 
The use of high performance composite fibers allows for the improvement of the mechanical properties of cement composites, which are determined predominantly by the interface properties between the fiber and cementitious matrix. Studies on the interface bond between fiber and cementitious matrix are discussed. The results show improvement on the compressive strength according to the embedment length of the fibers; moreover, chemical bond strength was independent of the water-to-binder ratio. Other studies cover experimental and analytical research results for predicting the flexural capacity of reinforced concrete (RC) beams strengthened in flexure with fabric reinforced cementitious matrix (FRCM). It was confirmed that the slippage between the FRP fabric and matrix occurs at a high strain level.

Unreinforced masonry (URM) structures represent a significant portion of existing historical structures around the world. Recent earthquakes have shown the need for seismic retrofitting for URM structures. A strengthening technique using externally bonded (EB) fiber-reinforced polymer (FRP) composites has attracted engineers since enhancing the shear strength of URM walls with negligible change to crosssectional area and weight of the walls. Results show that FRP composites were effective to increase shear strength. Confining of damaged or corroded concrete columns with FRP sheets is always interesting for researches. However, the performance of this strengthening system at elevated temperature is still questionable. The effects of elevated temperatures on the compressive strength capacity of carbon and glass FRP-confined concrete cylinders are shown.

Experimental investigation on failure behavior of the notched concrete beam strengthened with carbon fiber reinforced polymer (CFRP) is studied, including the influences of the length, thickness, and methods of bonding of CFRP laminate on the ultimate bearing capacity and the failure modes of the reinforced beam. A comparison between a theoretical analysis and the experimental observation shows that the failure modes observed in the experimental study are in agreement with the analytically predicted failure modes. another research is concerned with the effects of hooked-end steel fiber contents on the mechanical properties of high performance concrete (HPC) and investigates the feasibility of utilizing steel fibers to simplify the complicated reinforcement detailing of critical HPC members under high shear stress. It is also recommended that both stirrups and steel fiber should be used for fully confining the diagonal bar groups of coupling beams to achieve the ductile behavior.

Gonzalo Martínez-Barrera

Osman Gencel

João M. L. Reis 

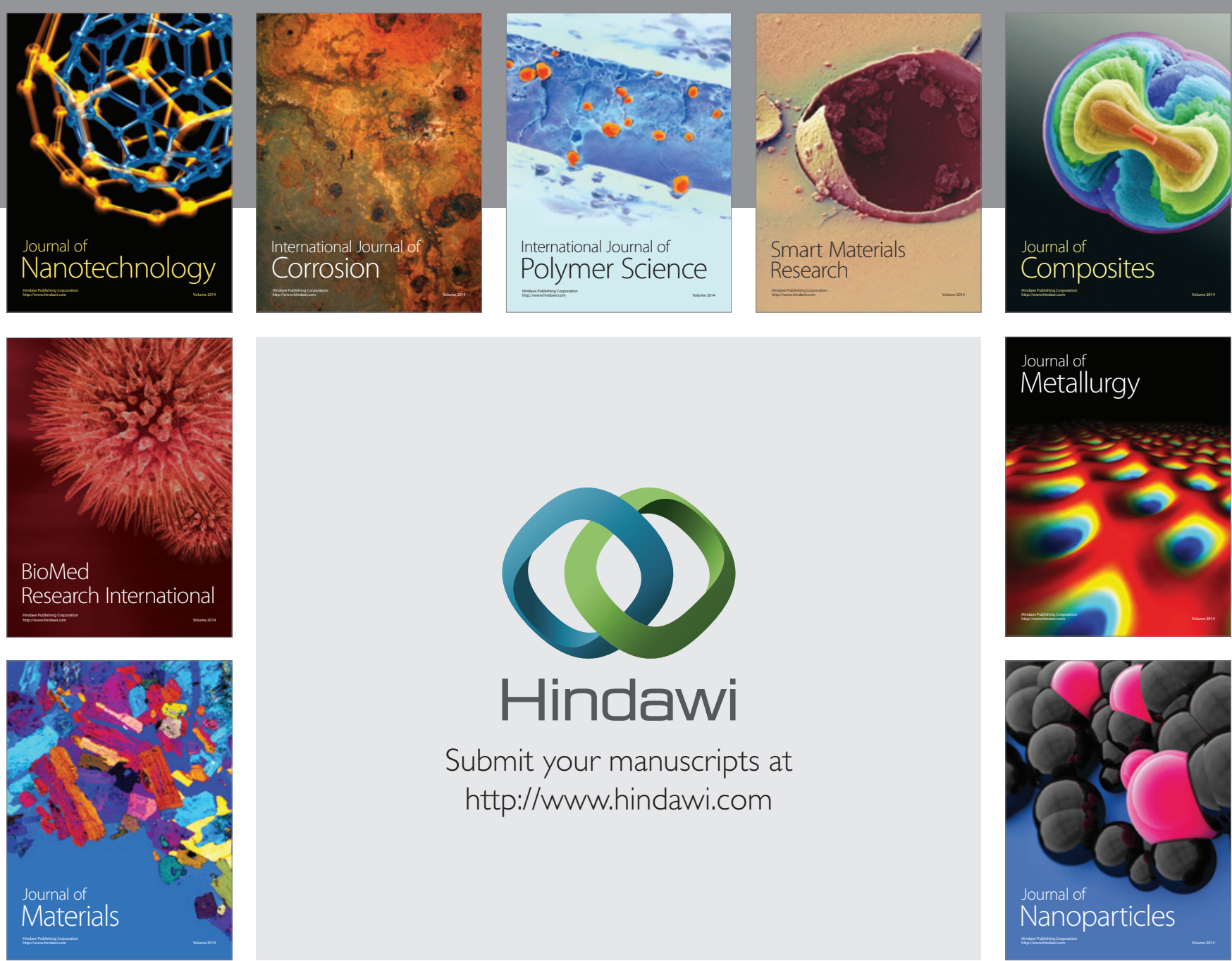

\section{Hindawi}

Submit your manuscripts at

http://www.hindawi.com

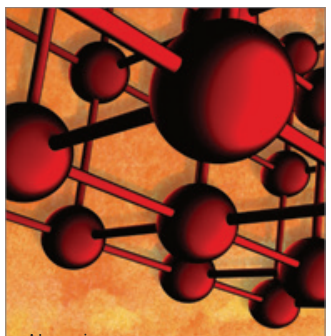

Materials Science and Engineering
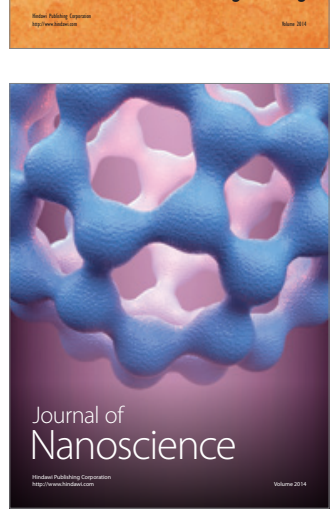
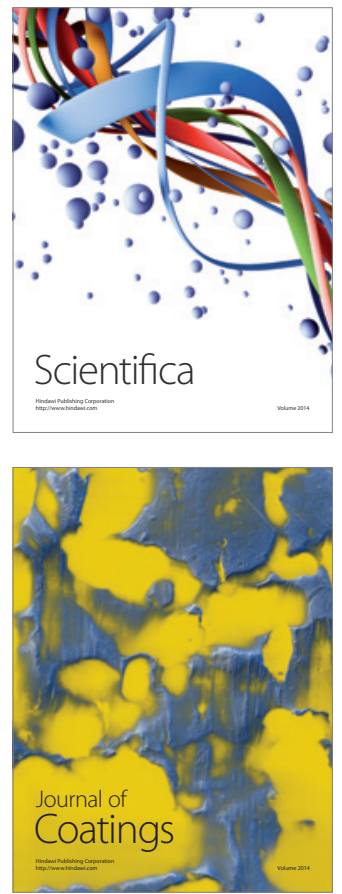
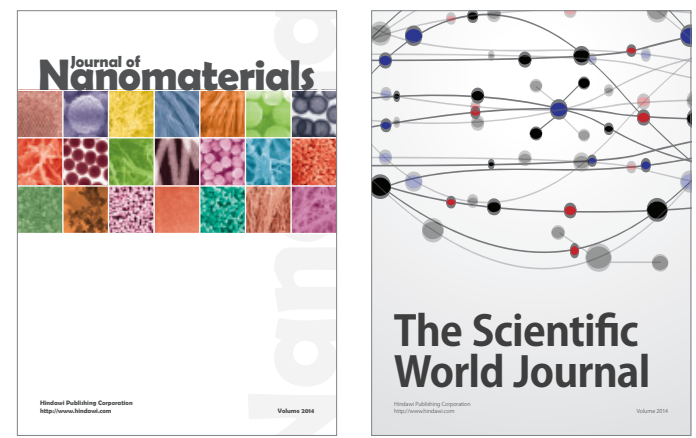

The Scientific World Journal
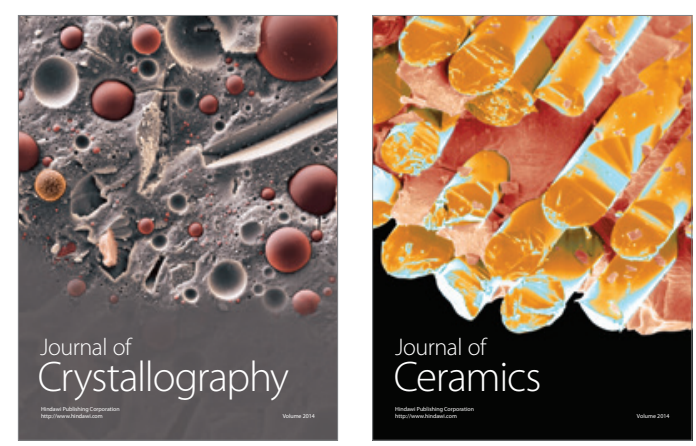
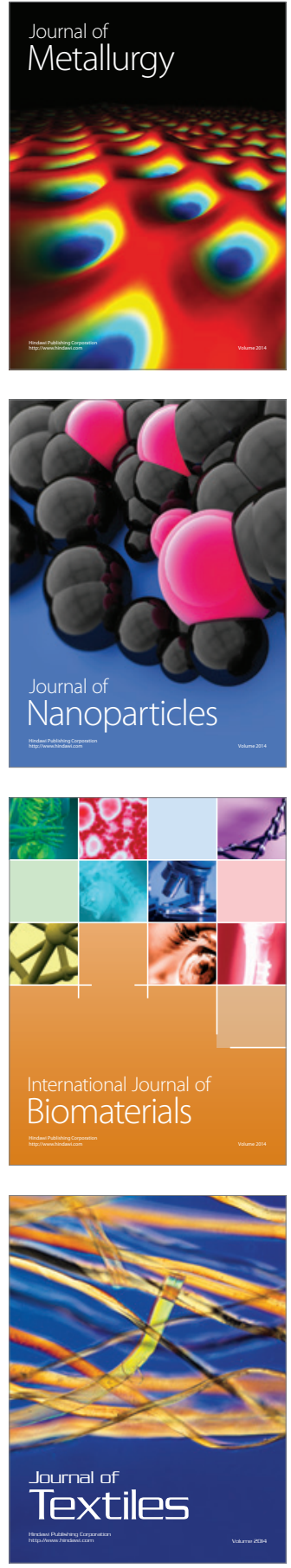